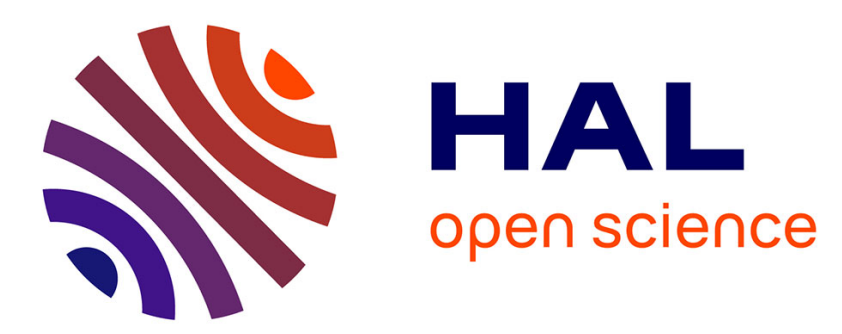

\title{
Fracture of disordered solids in compression as a critical phenomenon: I. Statistical mechanics formalism
}

Renaud Toussaint, Steven R Pride

\section{To cite this version:}

Renaud Toussaint, Steven R Pride. Fracture of disordered solids in compression as a critical phenomenon: I. Statistical mechanics formalism. Physical Review E: Statistical, Nonlinear, and Soft Matter Physics, 2002, 66 (3), pp.036135. 10.1103/physreve.66.036135 . hal-00110576

\section{HAL Id: hal-00110576 https://hal.science/hal-00110576}

Submitted on 20 Nov 2018

HAL is a multi-disciplinary open access archive for the deposit and dissemination of scientific research documents, whether they are published or not. The documents may come from teaching and research institutions in France or abroad, or from public or private research centers.
L'archive ouverte pluridisciplinaire HAL, est destinée au dépôt et à la diffusion de documents scientifiques de niveau recherche, publiés ou non, émanant des établissements d'enseignement et de recherche français ou étrangers, des laboratoires publics ou privés. 


\title{
Fracture of disordered solids in compression as a critical phenomenon. I. Statistical mechanics formalism
}

\author{
Renaud Toussaint* and Steven R. Pride ${ }^{\dagger}$ \\ Géosciences Rennes, Université de Rennes 1, 35042 Rennes Cedex, France
}

(Received 14 November 2001; revised manuscript received 13 June 2002; published 27 September 2002)

\begin{abstract}
This is the first of a series of three articles that treats fracture localization as a critical phenomenon. This first article establishes a statistical mechanics based on ensemble averages when fluctuations through time play no role in defining the ensemble. Ensembles are obtained by dividing a huge rock sample into many mesoscopic volumes. Because rocks are a disordered collection of grains in cohesive contact, we expect that once shear strain is applied and cracks begin to arrive in the system, the mesoscopic volumes will have a wide distribution of different crack states. These mesoscopic volumes are the members of our ensembles. We determine the probability of observing a mesoscopic volume to be in a given crack state by maximizing Shannon's measure of the emergent-crack disorder subject to constraints coming from the energy balance of brittle fracture. The laws of thermodynamics, the partition function, and the quantification of temperature are obtained for such cracking systems.
\end{abstract}

DOI: 10.1103/PhysRevE.66.036135

\section{INTRODUCTION}

When rocks and other disordered-solid materials are in compression and then have an additional deviatoric strain applied to them, small stable cracks irreversibly appear at random throughout the material. Each time the deviatoric strain is increased, more cracks appear. In the softening regime following peak stress, a sample will unstably fail along a plane localized at an angle relative to the principal-stress direction. We have accumulated evidence suggesting that such localization is a continuous phase transition.

This is the first of three articles that develops a statistical mechanics that allows the possible phase transitions in a cracking solid to be investigated. Many studies have assumed that, fracture is a thermally-activated process and have used a statistical mechanics based on thermal fluctuations [1-5]. However, our interest here is with "brittle fracture" in which cracks appear irreversibly and in which thermal fluctuations play no role. For this problem, the statistics of the fracture process is entirely due to the initial quenched disorder in the system.

A considerable literature has developed for so-called "breakdown" phenomena in systems having quenched disorder and zero temperature [6-23]. In particular, the burnedfuse [6-8], spring-network [9-11] and fiber-bundle [12-17] analog models for fracture have all been shown to yield various types of scaling laws prior to the point of breakdown [18-23]. Our work is different in that we directly treat the fracture problem (not an analog model of it) assuming that all of the statistics is due to quenched disorder. We obtain the probability of emergent damage states by maximizing Shannon's entropy subject to appropriate constraints. This ap-

\footnotetext{
*Present address: Department of Physics, University of Oslo, P.O. Box 1048 Blindern, 0316 Oslo 3, Norway. Email address: Renaud.Toussaint@fys.uio.no

†Email address: Steve.Pride@univ-rennes1.fr
}

proach has recently been proven exact in the special case of fiber bundles [24].

The principal conclusion of our present theory is that at a critical-strain point, there is a continuous phase transition from states where cracks are uniformly distributed to states where coherently oriented cracks are grouped into conjugate bands. Several facts justify classifying such band formation as a critical phenomenon.

First, the localization of the cracks into bands spontaneously breaks both the rotational and translational symmetries of the material even though our model Hamiltonian preserves these same symmetries. The entropy of the material remains continuous and the ensemble of the most probable states becomes degenerate at the localization transition; i.e., prior to localization, the most probable state is the intact state, while right at the transition, certain banded states acquire the same probability as the intact state. Further, an autocorrelation length associated with the aspect ratio of the emergent-crack bands diverges in the approach to the critical point. Unfortunately, quantitative laboratory measurements of how the bands of cracks coalesce and evolve in size and shape prior to the final localization point do not presently exist. We speculate in the third article of this series on how such measurements might be performed.

Our explanation of localization based on the physics of interacting cracks is distinct from the bifurcation analysis of Rudnicki and Rice [25] in which localization is a consequence of a proposed phenomenological elasto-plasticity law. Our work provides a method for obtaining such a plasticity law from the underlying physics.

\section{THE PROBABILISTIC NATURE OF THE FRACTURE PROBLEM}

Rocks are a disordered collection of grains in cohesive contact. The grains have varying shapes and sizes with typical grain sizes in the range of $10-100 \mu \mathrm{m}$ but sometimes considerably larger. The contacts between the grains are generally weaker than the grains themselves and have strengths and geometries that vary from one contact to the next. When deviatoric (i.e., shear) strain is applied to a rock, grain con- 
tacts begin to break. In what follows, a broken grain contact will be called a "crack." Such a break is a stress-activated irreversible process. Once a grain contact is broken, there is no significant healing that occurs. Cracks are not arriving and disappearing due to thermal fluctuations. This fact makes our definition of statistical ensembles quite different from that in the usual application of statistical mechanics to molecular systems, as we now go on to discuss.

\section{A. Creating a statistical ensemble}

We imagine dividing a huge (formally infinite) system into mesoscopic volumes that will be called "mesovolumes." Because the materials of interest here have a wide range of grain-scale disorder, many different crack states will emerge in the various mesovolumes once energy has been put into the system and cracking begins. These various mesovolumes and the crack states they contain comprise the ensembles in our theory.

In order to be specific with our ideas, we now introduce a simple model of the initial disorder and emergent-crack states. The purpose of this special model in the present paper is to motivate how ensembles are formed; however, the model Hamiltonian developed in Paper II will be based upon it.

In the model, each mesovolume is divided into $N$ identical cells, where a cell has dimensions on the order of a grain size and where $N$ is a large number such as $10^{2 D}$ or more with $D$ the system's dimension. In each cell, only a single grain contact is allowed to break. The local order parameter (explicitly defined in Paper II) characterizes both the orientation and the length of such a broken grain contact. In the present paper, an order-parameter description is not yet necessary. Prior to breaking, all cells are assumed to have the same elastic moduli.

The quenched disorder is in how the grain-contact breaking energy $\mathcal{E}(\boldsymbol{x})$ is distributed in the cells $\boldsymbol{x}$ of a mesovolume. We assume that only a fraction of the nominal grain-contact area is actually cemented together, and that the degree of cementation from one contact to the next is random. Thus, the breaking energies $\mathcal{E}(\boldsymbol{x})$ are random variables independently sampled from a distribution $\pi(\mathcal{E})$ having support on $\left[0, \Gamma d^{D-1}\right]$ where $\Gamma$ is the surface-energy density of the mineral, $d$ is the nominal linear dimension of a grain contact, and $d^{D-1}$ is the grain-contact area in $D$ dimensions. The quenched-disorder distribution $\pi(\mathcal{E})$ can have any assumed form.

We now define an infinite collection of distinct mesovolumes by allowing for every conceivable way that $\mathcal{E}(\boldsymbol{x})$ may be distributed in a mesovolume. Putting this collection together forms the infinite rock mass whose properties we are interested in determining. Each mesovolume so defined is a deterministic system and upon slowly applying the same strain tensor $\boldsymbol{\varepsilon}$ to all the mesovolumes, each will undergo a deterministic cracking scenario and end up in a well-defined crack state. We denote each of the possible final crack states with an index $j$. A principal goal of the present paper is to obtain the occupation probabilities $p_{j}$ of these various crack states that are simply the fraction of the mesovolumes in the system that are in the state $j$.

We can understand how the various crack states emerge by appealing to a form of Griffith's [26] criterion. A cell will break only if the change in the elastic energy due to the break is greater than or equal to the bond-breaking energy $\mathcal{E}(\boldsymbol{x})$. If $\mathbf{C}_{a}$ is the effective elastic-stiffness tensor of the entire mesovolume that holds after the break occurs and if $\mathbf{C}_{b}$ is the stiffness tensor that held before the break, Griffith's criterion can be stated,

$$
\ell^{D} \boldsymbol{\varepsilon}:\left(\mathbf{C}_{b}-\mathbf{C}_{a}\right): \boldsymbol{\varepsilon} / 2>\mathcal{E},
$$

where $\varepsilon$ is the strain tensor characterizing the entire mesovolume at the moment of the break and $\ell^{D}$ is the volume of a mesovolume. This particular statement is an approximation based on an assumed linear elasticity and absence of residual strain after unloading, but a general statement will be derived in Sec. III B. Since the mesovolume with an extra crack is more compliant than without it, the weakest cells will begin to break even after the slightest of applied strain.

Yet an emergent-crack state is not just a trivial consequence of the $\mathcal{E}(\boldsymbol{x})$ distribution in a mesovolume. Cracks aligned along bands concentrate stress allowing even large barriers $\mathcal{E}(\boldsymbol{x})$ to be overtaken along the band. In the present model, this means that placing cracks along bands produces a larger change in the elastic moduli of the mesovolume than placing cracks in more random positions. Thus, at least above some applied strain level, we expect the banded states to emerge as the ones that are significantly present in a rock system. Nonbanded states at large strain are much more special. They can come only from mesovolumes in which the weak cells making up the state are all surrounded by strong cells.

A key idea here is that each mesovolume embedded in the system experiences the same global strain tensor and, as such, has a crack state statistically independent from the other mesovolumes. This is only valid so long as the emergent bands of organized cracks have a dimension $\xi$ that is small relative to the size $\ell$ of the mesovolume. Screening effects due to destructive strain interactions between incoherently oriented cracks cause the far-field strain from a local crack structure to fall off with distance $r$ even more rapidly than the $(\xi / r)^{D}$ fall off in an uncracked material. But even in the thermodynamic limit of infinite system sizes, the required statistical independence of the mesovolumes breaks down right at the critical strain where divergent bands of cracks become important. The conclusion is that although our ensemble-based statistics is valid in the approach to localization, it is incapable of describing the post-localization physics.

\section{B. Macroscopic observables}

In the laboratory experiments to which we apply our theory, a sample is immersed in a reservoir from which either uniform stress or strain conditions can be applied to the sample's exterior surface $\partial \Omega$. The macroscopic strain tensor $\boldsymbol{\varepsilon}$ is defined in terms of the displacement $\mathbf{u}$ at points on $\partial \Omega$ as 


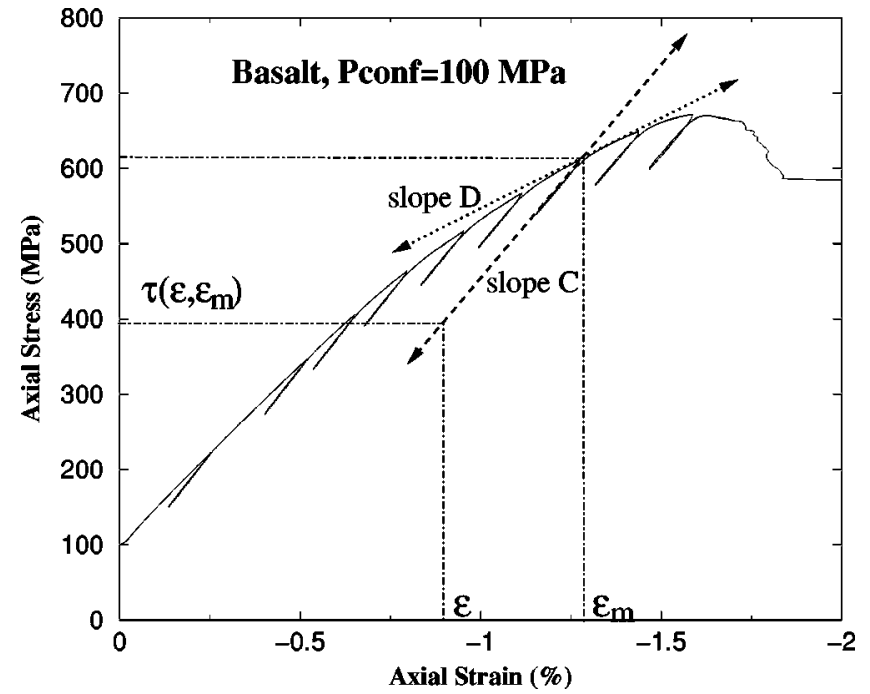

FIG. 1. Stress-strain data courtesy of David Lockner of the USGS Menlo Park. The slope measured upon loading a sample is defined by $\mathbf{D}$ while that measured upon unloading and/or reloading the sample is defined by $\mathbf{C}$.

$$
\boldsymbol{\varepsilon}=\frac{1}{L^{D}} \int_{\partial \Omega} \mathbf{n u} d S
$$

where $\mathbf{n}$ is the outward normal to the sample's surface and $L^{D}$ is the volume of the sample in $D$ dimensions. This definition of deformation thus corresponds to the volume average of the local deformation tensor $\boldsymbol{\nabla} \mathbf{u}(\boldsymbol{x})$ defined at interior points $\boldsymbol{x}$ of the sample. It will soon be shown to be conjugate to the macroscopic stress tensor $\boldsymbol{\tau}$ in the expression for the work carried out on the sample. If strain (rather than stress) is the control variable, the displacements at points $\boldsymbol{x}$ of the external surface $\partial \Omega$ are given by $\mathbf{u}=\boldsymbol{x} \cdot \boldsymbol{\varepsilon}$.

As shown in Fig. 1, a typical compression experiment starts with the sample in a pure hydrostatic pressure state and then systematically increases the deformation in the axial direction, keeping the radial "confining" pressure $p_{c}$ constant. Other ways of controlling the radial stress during the experiment are to keep a constant ratio between axial and radial stress, or to impose a constant radial deformation. So long as the confining pressure does not become so large as to induce a brittle-to-ductile transition [28], these various experiments all result in the same type of localized structure at large axial strains. When axial strain is monotonically increased, cracks arrive at each strain increment and the deformation and stress changes are related as

$$
d \boldsymbol{\tau}=\frac{d \boldsymbol{\tau}}{d \boldsymbol{\varepsilon}}: d \boldsymbol{\varepsilon}=\mathbf{D}: d \boldsymbol{\varepsilon}
$$

where the fourth-order tensor $\mathbf{D}$ is called the tangent-stiffness tensor. This tensor defines the slopes between the various stress and strain components as the sample is being loaded and is an experimental observable.

If at some point in the stress history the axial pressure is reduced, we follow a different deformation path as seen in the figure due to the fact that no new cracks are created. Such an unloading experiment defines the elastic (or secant) stiffness tensor $\mathbf{C}$,

$$
d \boldsymbol{\tau}=\mathbf{C}: d \boldsymbol{\varepsilon}
$$

We model the unloading/reloading paths as being entirely reversible and in so doing neglect the small hysteresis due to friction along the opened cracks.

In order to distinguish loading paths (with crack creation) from unloading paths (without crack creation), all properties are explicitly taken to depend on two strain variables; namely, the maximum strain $\boldsymbol{\varepsilon}_{m}$ having been applied to a sample, and the current strain $\boldsymbol{\varepsilon}$ that is different than the maximum only if the sample has been subsequently unloaded. Note that even if $\boldsymbol{\varepsilon}$ and $\boldsymbol{\varepsilon}_{m}$ are written as tensors, they each correspond to only one scalar degree of freedom along the loading/unloading paths, since the radial components can always be expressed in terms of the axial components via the type of radial control employed (e.g., $p_{c}$ $=$ const in a standard triaxial test).

The stress tensor $\boldsymbol{\tau}$ corresponds to the volume average of the local stress tensor $\mathbf{T}(\boldsymbol{x})$ that satisfies $\boldsymbol{\nabla} \cdot \mathbf{T}(\boldsymbol{x})=\mathbf{0}$ at interior points $\boldsymbol{x}$; i.e., $\boldsymbol{\tau}=L^{-D} \int_{\Omega} \mathbf{T}(\boldsymbol{x}) d V$ and is a function of the current and maximum strains $\boldsymbol{\tau}=\boldsymbol{\tau}\left(\boldsymbol{\varepsilon}, \boldsymbol{\varepsilon}_{m}\right)$ as shown in Fig. 1. By averaging the elastostatic identity $\boldsymbol{\nabla} \cdot(\mathbf{T} \boldsymbol{x})=\mathbf{T}$ over the mesovolume we further have that $\boldsymbol{\tau}=L^{-D} \int_{\partial \Omega} \mathbf{n} \cdot \mathbf{T} \boldsymbol{x} d S$.

The work density $d U$ performed on the sample when there is an increment in strain $d \boldsymbol{\varepsilon}$ is in both cases of loading and unloading

$$
\begin{aligned}
d U & =\frac{1}{L^{D}} \int_{\partial \Omega} \mathbf{n} \cdot \mathbf{T} \cdot d \mathbf{u} d S \\
& =\boldsymbol{\tau}: d \boldsymbol{\varepsilon} .
\end{aligned}
$$

To obtain Eq. (6) from (5), we have written the controlled displacements on a sample's surface as $d \mathbf{u}=\boldsymbol{x} \cdot d \boldsymbol{\varepsilon}$ where the strain increment $d \boldsymbol{\varepsilon}$ is uniform over $\partial \Omega$. Thus, $d U$ corresponds to the volume average of the local work density $\mathbf{T}(\boldsymbol{x}): d \boldsymbol{\nabla} \mathbf{u}(\boldsymbol{x})$.

The total energy $U$ per unit sample volume that goes into the sample during the loading up to a maximum strain tensor $\boldsymbol{\varepsilon}_{m}$ is then

$$
U\left(\boldsymbol{\varepsilon}_{m}\right)=\int_{\varepsilon_{0}}^{\varepsilon_{m}} \boldsymbol{\tau}\left(\boldsymbol{\varepsilon}^{\prime}, \boldsymbol{\varepsilon}^{\prime}\right): d \boldsymbol{\varepsilon}^{\prime},
$$

where $\varepsilon_{0}$ is the strain associated with the initial isotropic stress. If after loading to $\boldsymbol{\varepsilon}_{m}$, the sample is unloaded back to a current strain of $\boldsymbol{\varepsilon}$, we have the general expression

$$
U\left(\boldsymbol{\varepsilon}, \boldsymbol{\varepsilon}_{m}\right)=U\left(\boldsymbol{\varepsilon}_{m}\right)+\int_{\boldsymbol{\varepsilon}_{m}}^{\boldsymbol{\varepsilon}} \boldsymbol{\tau}\left(\boldsymbol{\varepsilon}^{\prime}, \boldsymbol{\varepsilon}_{m}\right): d \boldsymbol{\varepsilon}^{\prime}
$$

If the sample is unloaded back to the initial stress, corresponding to a possibly nonzero residual strain $\boldsymbol{\varepsilon}^{\text {res }}$, a last experimental observable is the energy $Q\left(\boldsymbol{\varepsilon}_{m}\right)=U\left(\boldsymbol{\varepsilon}^{\mathrm{res}}, \boldsymbol{\varepsilon}_{m}\right)$ 
(per unit sample volume) that went into crack creation and that is lost during the loading process .

\section{Ergodic hypothesis}

We have shown above that the experimentally measurable variables of energy density $U$, deformation $\boldsymbol{\varepsilon}$, and applied stress $\boldsymbol{\tau}$ correspond to volume averages of each field throughout a system. Our ergodic hypothesis amounts to assuming that the systems we work with are sufficiently large that such volume averages can be replaced by ensemble averages

$$
U=\sum_{j} p_{j} E_{j}, \quad \boldsymbol{\varepsilon}=\sum_{j} p_{j} \boldsymbol{\varepsilon}_{j}, \quad \boldsymbol{\tau}=\sum_{j} p_{j} \boldsymbol{\tau}_{j}
$$

Here, $E_{j}$ is the average work per unit mesovolume required to take an initially uncracked mesovolume from zero strain to the strain tensor $\boldsymbol{\varepsilon}_{j}$. A similar definition holds for $\boldsymbol{\tau}_{j}$. In both the definition of $E_{j}$ and $\boldsymbol{\tau}_{j}=d E_{j} / d \boldsymbol{\varepsilon}_{j}$, the average is over the initial quenched-disorder distribution.

So long as each mesovolume contains crack states that have no significant influence on the neighboring mesovolumes (formally valid only in the thermodynamic limit), the sum over the collection of mesovolumes (ensemble averaging) is equivalent to a volume integral over the entire system. In practice, we will only ever consider ensembles that have by definition $\boldsymbol{\varepsilon}_{j}=\boldsymbol{\varepsilon}$; however, we could equivantly immerse each mesovolume in a uniform stress-tensor reservoir and allow $\boldsymbol{\varepsilon}_{j}$ to vary from state to state.

\section{THERMODYNAMICS OF CRACK POPULATIONS}

\section{A. Fundamental postulate}

The fracture-mechanics problem of counting how many of the initial mesovolumes can be led to the same crack state appears to be hopelessly intractable. Fortunately, it also appears to be unnecessary for systems containing initial quenched disorder. Upon putting deviatoric strain energy into such a system, the emergent-crack states $j$ will, on the one hand, attempt to mirror this quenched disorder with weakest cells breaking first; however, due to the energetics of the crack interactions, many different types of initial mesovolumes may be led to the same crack state which results in nonuniform crack-state probabilities $p_{j}$ even if the quencheddisorder distribution is uniform.

We state our fundamental postulate as follows: The probability $p_{j}$ of observing a mesovolume to be in crack state $j$ can be determined by maximizing Shannon's [27] measure of disorder

$$
S=-\sum_{j} p_{j} \ln p_{j}
$$

subject to constraints involving the macroscopic observables that derive from the energetics of the fracture mechanics. That entropy is to be maximized can be expected since the quenched disorder allows all states to be present in a sufficiently large system. In recent work [24], we have demon-

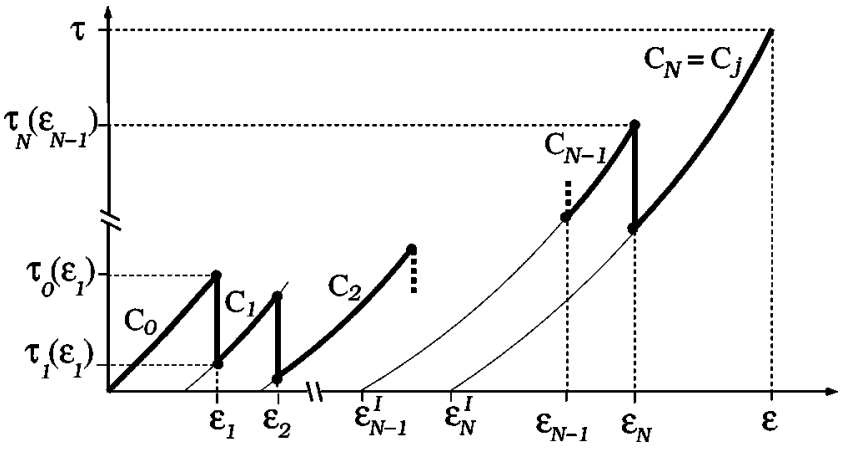

FIG. 2. The heavy line is the actual path followed during the steady application of axial strain. Each vertical drop in stress corresponds to the arrival of a crack.

strated that this postulate yields exact results for the special case of fiber bundles with global-load sharing.

The constraints are what give the dimensionless function $S$ defined by Eq. (10) all the thermodynamic information about our cracking system and must explicitly involve the independent variables of $S$. Such independent variables are determined by establishing the first law of thermodynamics for a system cracking in compressive shear.

\section{B. The work of creating a crack state}

To obtain the first law, it is first necessary to define the detailed energy balance for each crack state and to understand how the work $E_{j}$ required to create state $j$ depends on both the actual strain $\varepsilon$ and on the maximum-achieved strain $\boldsymbol{\varepsilon}_{m}$.

\section{Griffith's criterion and crack-state energy}

Consider a given mesovolume with a deterministic distribution of breaking energies $\mathcal{E}(\boldsymbol{x})$ assigned to each cell $\boldsymbol{x}$ of the mesovolume. Starting from a state of isotropic strain $\boldsymbol{\varepsilon}_{0}$, we slowly apply an additional axial deformation and monitor how one crack after another enters the mesovolume until the final strain tensor $\boldsymbol{\varepsilon}$ and final crack state $j$ are arrived at. Lets say that this state $j$ has a total of $\mathcal{N}$ cracks associated with it.

Figure 2 details the history of how the stress (and, therefore, work) might evolve in the mesovolume as strain is applied and cracks arrive. Initially, the mesovolume will elastically deform according to the stiffness tensor $\mathbf{C}_{0}$ (no cracks yet present) until the first crack arrives at the strain tensor $\boldsymbol{\varepsilon}_{1}$ with an associated drop in the mesovolume's stress. Lets say the bond-breaking energy of this first crack was $\mathcal{E}_{1}$. The mesovolume will now have a different overall stiffness tensor $\mathbf{C}_{1}$ and will elastically deform with these new moduli until the second crack arrives and so on until all $\mathcal{N}$ cracks have entered and the mesovolume has attained its final stiffness tensor of $\mathbf{C}_{j}=\mathbf{C}_{\mathcal{N}}$. The final tensor $\mathbf{C}_{j}$ depends on both the location and orientation of these $\mathcal{N}$ cracks in addition to their number.

At some intermediate stage having $n$ cracks, the stress tensor $\boldsymbol{\tau}_{n}(\boldsymbol{\varepsilon})$ is defined by integrating $d \boldsymbol{\tau}=\mathbf{C}_{n}\left(\boldsymbol{\varepsilon}^{\prime}\right): d \boldsymbol{\varepsilon}^{\prime}$ from $\boldsymbol{\varepsilon}_{n}^{\text {res }}$ to $\boldsymbol{\varepsilon}$, where $\boldsymbol{\varepsilon}_{n}^{\text {res }}$ is the "residual" deformation observed upon unloading the sample back to zero stress as shown in the figure. We have 


$$
\boldsymbol{\tau}_{n}(\boldsymbol{\varepsilon})=\int_{\varepsilon_{n}^{\mathrm{res}}}^{\boldsymbol{\varepsilon}} \mathbf{C}_{n}: d \boldsymbol{\varepsilon}^{\prime}
$$

The elastic energy density corresponding to this state at deformation $\boldsymbol{\varepsilon}$ is similarly

$$
E_{n}^{\mathrm{el}}(\boldsymbol{\varepsilon})=E_{n}^{\mathrm{res}}+\int_{\varepsilon_{n}^{\mathrm{res}}}^{\boldsymbol{\varepsilon}} \boldsymbol{\tau}_{n}\left(\boldsymbol{\varepsilon}^{\prime}\right): d \boldsymbol{\varepsilon}^{\prime},
$$

where $E_{n}^{\text {res }}$ represents the residual elastic energy that remains in the system when the state with $n$ cracks is unloaded to zero applied stress. These residual (zero stress) quantities are present whenever plastic deformation occurs within a grain contact. After a sample elastically returns to zero applied stress, such plastic deformation remains and, accordingly, there is an elastic stress field surrounding any crack that experienced plastic deformation. The strain energy associated with such local residual stress is what constitutes the residual energy $E_{n}^{\text {res }}$.

When the $n$th crack arrives in a strain-controlled experiment, there is no change in the strain $\boldsymbol{\varepsilon}_{n}$ and thus no external work performed. However, there is a change in stiffness (and possibly residual strain) resulting in an associated stress drop $\Delta \boldsymbol{\tau}_{n}=\boldsymbol{\tau}_{n-1}\left(\boldsymbol{\varepsilon}_{n}\right)-\boldsymbol{\tau}_{n}\left(\boldsymbol{\varepsilon}_{n}\right)$, and a drop in the stored elastic energy density $\Delta E_{n}^{\mathrm{el}}=E_{n-1}^{\mathrm{el}}\left(\boldsymbol{\varepsilon}_{n}\right)-E_{n}^{\mathrm{el}}\left(\boldsymbol{\varepsilon}_{n}\right)$. Energy conservation requires the elastic energy reduction to exactly balance the work performed in opening the crack so that

$$
-\Delta E_{n}^{\mathrm{el}}+\frac{\mathcal{E}_{n}+K_{n}}{\ell^{D}}=0
$$

where $\mathcal{E}_{n}$ is the bond-breaking work performed at the grain contact of the $n$th crack, $K_{n}$ is the energy that went into acoustic emissions when the crack arrived and/or expended in any mode II frictional sliding or plastic deformation at the grain contact ( $K_{n}$ is a positive "loss" term), and, as earlier, $\ell^{D}$ is the volume of a mesovolume. Because $K_{n}$ is positive, we can rewrite Eq. (13) as an inequality

$$
\frac{K_{n}}{\ell^{D}}=\Delta E_{n}^{\mathrm{el}}-\frac{\mathcal{E}_{n}}{\ell^{D}} \geqslant 0,
$$

which is a general statement of Griffith's criterion. Upon appealing to linear elasticity (elastic stiffnesses independent of strain level) and putting the residual deformation to zero (no plasticity inside the cracks), we arrive at the convenient statement $\ell^{D} \boldsymbol{\varepsilon}_{n}:\left(\mathbf{C}_{n-1}-\mathbf{C}_{n}\right): \boldsymbol{\varepsilon}_{n} / 2 \geqslant \mathcal{E}_{n}$ given earlier.

The work performed between the arrival of the $n$th and the $(n+1)$ th crack is defined,

$$
W_{n}=\int_{\varepsilon_{n}}^{\boldsymbol{\varepsilon}_{n+1}} \boldsymbol{\tau}_{n}\left(\boldsymbol{\varepsilon}^{\prime}\right): \boldsymbol{\varepsilon}^{\prime}=E_{n}^{\mathrm{el}}\left(\boldsymbol{\varepsilon}_{n+1}\right)-E_{n}^{\mathrm{el}}\left(\boldsymbol{\varepsilon}_{n}\right) .
$$

Thus, the total work required to reach the final strain $\boldsymbol{\varepsilon}$ is the sum (cf. Fig. 2)

$$
E_{j}^{p}=\sum_{m=0}^{\mathcal{N}} W_{n}
$$

where by convention $W_{\mathcal{N}}$ is the work performed after the arrival of the last crack to get to the final deformation $\boldsymbol{\varepsilon}$. The superscript $p$ on $E_{j}^{p}$ is simply indicating that this is the work for one particular realization of the quenched disorder. Rewriting the sum by introducing Eqs. (15) and (13), then gives

$$
\begin{aligned}
E_{j}^{p} & =E_{\mathcal{N}}^{\mathrm{el}}(\boldsymbol{\varepsilon})-E_{0}^{\mathrm{el}}\left(\boldsymbol{\varepsilon}_{0}\right)+\sum_{n=1}^{\mathcal{N}} \Delta E_{n}^{\mathrm{el}} \\
& =E_{j}^{\mathrm{el}}(\boldsymbol{\varepsilon})+\sum_{n=1}^{\mathcal{N}} \frac{\mathcal{E}_{n}+K_{n}}{\ell^{D}}-E_{0}^{\mathrm{el}}\left(\boldsymbol{\varepsilon}_{0}\right),
\end{aligned}
$$

where $E_{0}^{\mathrm{el}}\left(\boldsymbol{\varepsilon}_{0}\right)$ is the small and physically unimportant amount of energy that is stored in the initial isotropic strain field. Equation (17) is the natural statement that the work performed in creating state $j$ at strain $\boldsymbol{\varepsilon}$ is the sum of the elastic energy density stored in the material in the final state plus the energy irreversibly expended during the opening of each crack.

Both the loss term $K_{n}$ and the residual energies $E_{j}^{\text {res }}$ (contained in $E_{j}^{\mathrm{el}}$ ) are potentially a function of the point in strain history at which a grain contact actually breaks; e.g., most models one might propose for plastic deformation at a grain contact are dependent on the applied stress level. However, modeling such plastic processes seems uncertain at best. We thus assume that at least for those crack states significantly contributing to any phase transition (states with lots of cracks), the stress-history dependence of $K_{n}$ is, on average, negligible. Further, since the residual strain in brittle-fracture experiments is never more than a few percent of the peakstress deformation and since the essence of the localization process does not seem to lie in $E_{j}^{\text {res }}$, we assume that $E_{j}^{\text {res }}$ $\ll \Sigma_{n} \mathcal{E}_{n}$. With these approximations, the work density $E_{j}^{p}$ depends only on the final state $j$, the final strain $\boldsymbol{\varepsilon}$ (through $\left.E_{j}^{\mathrm{el}}\right)$, and the breaking energies $\mathcal{E}_{n}$.

The energy density $E_{j}$ needed later in our probability law is obtained by further averaging over the quenched disorder in the breaking energies $\mathcal{E}_{n}$ to give

$$
E_{j}=E_{j}^{\mathrm{el}}(\boldsymbol{\varepsilon})+\gamma_{j}\left(\boldsymbol{\varepsilon}_{m}\right) \frac{\mathcal{N}_{j}}{\ell^{D}}-E_{0}^{\mathrm{el}}\left(\boldsymbol{\varepsilon}_{0}\right) .
$$

Here, $\mathcal{N}_{j}=\mathcal{N}$ is the total number of cracks in state $j$ and $\gamma_{j}$ is the average energy required to break a single grain contact where the average is over all cells throughout all mesovolumes led to state $j$. This $\gamma_{j}$ can be different for different final crack states. It will also be greater at greater values of the maximum strain $\boldsymbol{\varepsilon}_{m}$ because, according to Griffith, the cells comprising $j$ can break at higher energy levels when the strain is greater. The first term in Eq. (18) corresponds to the purely reversible elastic energy and therefore depends only on the actual strain state $\boldsymbol{\varepsilon}$.

\section{Specific expression for $E_{j}$}

To facilitate the development in Paper II and to be more specific, we now use Griffith's criterion to develop an ex- 
pression for $E_{j}$ that is based on linear elasticity. When the $n$th crack arrives, the linear-elastic variant of the Griffith criterion gives that

$$
\begin{aligned}
\mathcal{E}_{n} & <\ell^{D} \boldsymbol{\varepsilon}_{n}:\left(\mathbf{C}_{n-1}-\mathbf{C}_{n}\right): \boldsymbol{\varepsilon}_{n} / 2 \\
& <\ell^{D} \boldsymbol{\varepsilon}_{m}:\left(\mathbf{C}_{n-1}-\mathbf{C}_{n}\right): \boldsymbol{\varepsilon}_{m} / 2,
\end{aligned}
$$

where as earlier $\boldsymbol{\varepsilon}_{n}$ is the strain point on the load curve where the $n$th crack arrives while $\boldsymbol{\varepsilon}_{m}$ is the final maximum strain level of the experiment. The second inequality follows from the first since an extra crack always reduces the stiffness of a mesovolume. For any particular mesovolume in state $j$, the average energy required to break a contact $\gamma_{j}^{p}$ thus satisfies

$$
\gamma_{j}^{p} \equiv \frac{1}{\mathcal{N}_{j}} \sum_{n=1}^{\mathcal{N}_{j}} \mathcal{E}_{n}<\frac{\ell^{D}}{2 \mathcal{N}_{j}} \boldsymbol{\varepsilon}_{m}:\left(\mathbf{C}_{0}-\mathbf{C}_{j}\right): \boldsymbol{\varepsilon}_{m},
$$

where the right-hand side comes from summing Eq. (20). Since this inequality is independent of the history, every mesovolume that is led to state $j$ must satisfy it. We may thus write $\gamma_{j}$ in the form

$$
\gamma_{j}=f_{j} \frac{\ell^{D}}{2 \mathcal{N}_{j}} \boldsymbol{\varepsilon}_{m}:\left(\mathbf{C}_{0}-\mathbf{C}_{j}\right): \boldsymbol{\varepsilon}_{m},
$$

where the fraction $f_{j}$ is bounded as $0<f_{j}<1$. We next demonstrate that the variation of $f_{j}$ from one state to the next is so small as to be neglected altogether.

A tighter lower bound for $f_{j}$ is obtained by considering crack states $j$ having $\mathcal{N}_{j}$ noninteracting cracks. Since the cracks do not interact to concentrate stress, all of the $\mathcal{N}_{j}$ cells that broke had their breaking energies somewhere in the range $0 \leqslant \mathcal{E} \leqslant \delta E=\ell^{D} \boldsymbol{\varepsilon}_{m}: \delta \mathbf{C}: \boldsymbol{\varepsilon}_{m} / 2$, where $\delta \mathbf{C}$ is the change in the stiffness tensor due to the arrival of a single noninteracting crack and $\delta E$ is the associated change in the elastic energy. Since the breaking energies are independent random variables taken from the distribution $\pi(\mathcal{E})$, we obtain

$$
\gamma_{j}=\frac{\int_{0}^{\delta E} e \pi(e) d e}{\int_{0}^{\delta E} \pi(e) d e}
$$

for noninteracting crack states $j$.

We now appeal to a specific form for the probability distribution $\pi(\mathcal{E})$. Initially, our rocks are intact and it is expected that more grain contacts are entirely bonded $(\mathcal{E}$ $\left.=\Gamma d^{D-1}\right)$ than entirely unbonded $(\mathcal{E}=0)$. We thus assume a monotonic distribution $\mathcal{E}^{k}$ with $k>0$ satisfying the normalization $\int_{0}^{\Gamma d^{D-1}} \pi(e) d e=1$ so that

$$
\pi(\mathcal{E})=\frac{(k+1)}{\Gamma d^{D-1}}\left(\frac{\mathcal{E}}{\Gamma d^{D-1}}\right)^{k}=c \mathcal{E}^{k}
$$

Using this $\pi$, the average energy required to break a contact in a noninteracting crack state is

$$
\gamma_{j}=\frac{k+1}{k+2} \delta E=\frac{q}{2} \ell^{D} \boldsymbol{\varepsilon}_{m}: \delta \mathbf{C}: \boldsymbol{\varepsilon}_{m},
$$

where we have defined $q=(k+1) /(k+2)$. All dependence on the underlying quenched-disorder distribution in our theory is confined to the constant $q$ which for any $k>0$ is in the range $[0.5,1]$.

Since for noninteracting states $\mathbf{C}_{0}-\mathbf{C}_{j}=\mathcal{N}_{j} \delta \mathbf{C}$, a comparison of Eqs. (25) and (22) shows that $f_{j}=q$ for all the noninteracting states. For the interacting states, the prefactor $f_{j}$ must be slightly greater because now stress concentration can allow stronger cells to break. It is thus concluded that for all states, the $f_{j}$ of Eq. (22) are bounded as $q \leqslant f_{j}<1$ which when compared to how $\mathcal{N}_{j}$ varies from state to state can be considered negligible. From here on, we simply take $f_{j}=q$ for all states.

The essential physics for the average amount of work that goes into building up any given crack state $j$ is thus captured by

$$
\begin{gathered}
E_{j}\left(\boldsymbol{\varepsilon}, \boldsymbol{\varepsilon}_{m}\right)=E_{j}^{R}(\boldsymbol{\varepsilon})+E_{j}^{I}\left(\boldsymbol{\varepsilon}_{m}\right), \\
E_{j}^{R}(\boldsymbol{\varepsilon})=\frac{1}{2} \boldsymbol{\varepsilon}: \mathbf{C}_{j}: \boldsymbol{\varepsilon}, \\
E_{j}^{I}\left(\boldsymbol{\varepsilon}_{m}\right)=\frac{q}{2} \boldsymbol{\varepsilon}_{m}:\left(\mathbf{C}_{0}-\mathbf{C}_{j}\right): \boldsymbol{\varepsilon}_{m},
\end{gathered}
$$

where the superscripts $R$ and $I$ denote respectively the reversible and irreversible part of the energy. The intact hydrostatic energy $E_{0}^{\mathrm{el}}\left(\boldsymbol{\varepsilon}_{0}\right)$ has been neglected since it does not involve cracks and, therefore, cannot influence the probability of the various crack states.

\section{The laws of our crack-based thermodynamics}

Using the ergodic hypothesis discussed earlier, the average energy density in a disorded solid can be written $U$ $=\Sigma_{j} p_{j} E_{j}$. We are interested in how $U$ changes when increments in $\boldsymbol{\varepsilon}$ and $\boldsymbol{\varepsilon}_{m}$ are applied to the system.

In general, a small increment in $U$ can be written as

$$
d U=\sum_{j} E_{j} d p_{j}+\sum_{j} p_{j} d E_{j}
$$

The first term involving the probability change is entirely due to crack creation. Some mesovolumes that were in less cracked states prior to the increment, are transformed to state $j$ during the increment, while mesovolumes that were in state $j$, are transformed to other more cracked states. If in the increment, the number of mesovolumes arriving in state $j$ is different than the number leaving, there is a change $d p_{j}$ in the occupational probability of that state. Such changes are the only way to change the disorder in the system, so that

$$
\sum_{j} E_{j} d p_{j}=T d S
$$


is the work involved in changing the system's disorder via crack production. The proportionality constant $T$ is formally a temperature and will be treated in detail.

Using the decomposition $E_{j}\left(\boldsymbol{\varepsilon}, \boldsymbol{\varepsilon}_{m}\right)=E_{j}^{R}(\boldsymbol{\varepsilon})+E_{j}^{I}\left(\boldsymbol{\varepsilon}_{m}\right)$, we can write the second term of Eq. (29) as

$$
\sum_{j} p_{j} d E_{j}=\sum_{j} p_{j} d E_{j}^{R}+\sum_{j} p_{j} d E_{j}^{I}
$$

The first part is due to purely elastic (reversible) changes in each mesovolume and may be further written

$$
\sum_{j} p_{j} d E_{j}^{R}=\boldsymbol{\tau}: d \boldsymbol{\varepsilon}
$$

where $\boldsymbol{\tau}$ is the average stress tensor acting on the mesovolumes. This result can be verified by appealing either to Eq. (27) or to the more general statement of Eq. (12).

The second part $\Sigma_{j} p_{j} d E_{j}^{I}$ represents the average work performed in creating cracks in just the final strain increment $d \boldsymbol{\varepsilon}_{m}$. Some of the initial mesovolumes led to state $j$ at maximum strain $\boldsymbol{\varepsilon}_{m}+d \boldsymbol{\varepsilon}_{m}$ had all their cracks in place before the final strain increment, while others had cracks arrive in the final increment. We write

$$
\sum_{j} p_{j} d E_{j}^{I}=\mathbf{g}: d \boldsymbol{\varepsilon}_{m}
$$

where the tensor $\boldsymbol{g}$ has units of stress but is quite distinct from the stress tensor $\boldsymbol{\tau}$.

The "first law" for the rock mass is then

$$
d U=\boldsymbol{\tau}: d \boldsymbol{\varepsilon}+\boldsymbol{g}: d \boldsymbol{\varepsilon}_{m}+T d S,
$$

with the formal definitions

$$
\boldsymbol{\tau}=\left.\frac{\partial U}{\partial \boldsymbol{\varepsilon}}\right|_{\boldsymbol{\varepsilon}_{m}, S}, \quad \boldsymbol{g}=\left.\frac{\partial U}{\partial \boldsymbol{\varepsilon}_{m}}\right|_{\boldsymbol{\varepsilon}, S}, \quad \text { and } \quad T=\left.\frac{\partial U}{\partial S}\right|_{\boldsymbol{\varepsilon}, \boldsymbol{\varepsilon}_{m}} .
$$

The natural variables of the fundamental function $U$ are $\left(S, \boldsymbol{\varepsilon}, \boldsymbol{\varepsilon}_{m}\right)$. Equivalently if $S$ is treated as the fundamental function, then $S=S\left(U, \boldsymbol{\varepsilon}, \boldsymbol{\varepsilon}_{m}\right)$ which means that the constraints placed on the maximization of $S$ must involve $U, \boldsymbol{\varepsilon}$, and $\boldsymbol{\varepsilon}_{m}$.

The "second law" of this crack-based thermodynamics is that $d S \geqslant 0$ (equal to zero only if $d \boldsymbol{\varepsilon}_{m}=0$ so that no cracks are created) while a "third law" may be proposed by simply defining $T=0$ when $S=0$. The system will have zero emergent disorder before cracks begin to arrive and so our third law states that the temperature $T$ starts at zero and then increases in magnitude as the number of cracks in the system increases from zero. The justification for this postulate comes a posteriori when it is found that in order to have zero probability for a mesovolume being in anything but the uncracked state $(S=0)$, we must have that $T=0$.

\section{The probability distribution}

To obtain the probability of observing a mesovolume to be in crack state $j$, we maximize $S=-\Sigma_{j} p_{j} \ln p_{j}$ subject to the constraint that $\Sigma_{j} p_{j}=1$, and to the additional constraints that $\boldsymbol{\varepsilon}_{j}=\boldsymbol{\varepsilon}, \boldsymbol{\varepsilon}_{m j}=\boldsymbol{\varepsilon}_{m}$, and $\sum_{j} p_{j} E_{j}=U$. These constraints define our canonical ensemble. Other ensembles can be defined by considering other constraints involving $\boldsymbol{\varepsilon}, \boldsymbol{\varepsilon}_{m}$, and $U$; however, since all ensembles yield identical average properties in the thermodynamic limit, we elect to work only with the canonical ensemble due to its analytical convenience.

This maximization problem is solved using Lagrange multipliers to obtain the Boltzmannian

$$
p_{j}=\frac{e^{-E_{j} / T}}{Z}, \quad \text { where } Z=\sum_{j} e^{-E_{j} / T},
$$

and where the parameter $T$ is exactly the partial derivative $\partial U /\left.\partial S\right|_{\boldsymbol{\varepsilon}, \boldsymbol{\varepsilon}_{m}}$ called "temperature."

\section{E. The free energy and its derivatives}

Any equilibrium physical property that depends on the distribution of cracks throughout the system can be obtained from the partition function $Z$ given by Eq. (36).

To do so, a thermodynamic potential $F$ called the freeenergy density is introduced that is related to $Z$ by

$$
F\left(\boldsymbol{\varepsilon}, \boldsymbol{\varepsilon}_{m}, T\right)=-T \ln Z\left(\boldsymbol{\varepsilon}, \boldsymbol{\varepsilon}_{m}, T\right) .
$$

This potential $F$ is the Legendre transform with respect to $S$ of the total-energy density $U=U\left(\boldsymbol{\varepsilon}, \boldsymbol{\varepsilon}_{m}, S\right)$ as can be seen from

$$
U-T S=\sum_{j} p_{j} E_{j}+T \sum_{j} p_{j} \ln p_{j}=-T \ln Z \sum_{j} p_{j}=F,
$$

where we used that $\ln p_{j}=-E_{j} / T-\ln Z$.

When $\left(\boldsymbol{\varepsilon}, \boldsymbol{\varepsilon}_{m}, T\right)$ are the independent variables, the first law can be obtained by taking the total derivative of Eq. (37)

$$
\begin{aligned}
d F & =-T \frac{d Z}{Z}-\ln Z d T \\
& =-T \sum_{j}\left[-\frac{d E_{j}\left(\boldsymbol{\varepsilon}, \boldsymbol{\varepsilon}_{m}\right)}{T}+E_{j} \frac{d T}{T^{2}}\right] p_{j}-\ln Z d T \\
& =(F-U) \frac{d T}{T}+\sum_{j} p_{j}\left[d E_{j}^{R}(\boldsymbol{\varepsilon})+d E_{j}^{I}\left(\boldsymbol{\varepsilon}_{m}\right)\right] \\
& =-S d T+\boldsymbol{\tau} \cdot d \boldsymbol{\varepsilon}+\boldsymbol{g}: d \boldsymbol{\varepsilon}_{m},
\end{aligned}
$$

where we have used the definitions that $\boldsymbol{\tau}_{j}=d E_{j}^{R}(\boldsymbol{\varepsilon}) / d \boldsymbol{\varepsilon}$ and $\boldsymbol{g}_{j}=d E_{j}^{I}\left(\boldsymbol{\varepsilon}_{m}\right) / d \boldsymbol{\varepsilon}_{m}$.

With $\beta=1 / T$, the various thermodynamic functions are related to the partial derivatives of $\ln Z\left(\boldsymbol{\varepsilon}, \boldsymbol{\varepsilon}_{m}, \beta\right)$ as

$$
-\frac{\partial \ln Z}{\partial \beta}=\sum_{j} E_{j} p_{j}=U,
$$




$$
\begin{gathered}
-\frac{1}{\beta} \frac{\partial \ln Z}{\partial \boldsymbol{\varepsilon}}=\sum_{j} \boldsymbol{\tau}_{j} p_{j}=\boldsymbol{\tau}, \\
-\frac{1}{\beta} \frac{\partial \ln Z}{\partial \boldsymbol{\varepsilon}_{m}}=\sum_{j} \boldsymbol{g}_{j} p_{j}=\boldsymbol{g} .
\end{gathered}
$$

These results, along with $S=\ln Z+\beta U$, are used in Paper III.

\section{TEMPERATURE}

The temperature is a well-defined essential part of our quenched-disorder statistics. Through the probability law $p_{j}$ $=e^{-E_{j} / T} / Z$, the temperature quantifies the energy scale that separates probable from improbable states and how this energy scale evolves with strain. No other meaning should be read into $T$. We now demonstrate how to exactly obtain $T$.

\section{A. Evolution of temperature with strain}

The only way energy enters the system is by performing work on the external surface. Thus, the general relation $d U$ $=\boldsymbol{\tau}: d \boldsymbol{\varepsilon}$ holds for either loading or unloading situations. This previously unused fact provides a differential equation for $T=1 / \beta$ that permits everything about our system to be exactly known once an order-parameter based model for $E_{j}\left(\boldsymbol{\varepsilon}, \boldsymbol{\varepsilon}_{m}\right)$ is determined and the functional sums defining $Z\left(\boldsymbol{\varepsilon}, \boldsymbol{\varepsilon}_{m}, \beta\right)=\Sigma_{j} e^{-\beta E_{j}\left(\boldsymbol{\varepsilon}, \boldsymbol{\varepsilon}_{m}\right)}$ are performed.

The temperature and entropy only evolve along load paths defined by $\boldsymbol{\varepsilon}=\boldsymbol{\varepsilon}_{m}$ and only such paths need be considered in what follows. Using $d U=\boldsymbol{\tau}: d \boldsymbol{\varepsilon}$, the first law [Eq. (34)] can then be rewritten as

$$
T d S+g: d \boldsymbol{\varepsilon}=0 .
$$

Since it always requires energy to break contacts, we have that $g: d \boldsymbol{\varepsilon}>0$ and consequently $T d S<0$. Furthermore, since the entropy (disorder) necessarily grows during the crackcreation process (at least initially), the temperature of our system is negative (at least initially).

The load path of a standard triaxial experiment is when axial strain $\varepsilon_{z}$ monotonically increases while the radial confining stress $\tau_{x}=\tau_{y}=-p_{c}$ remains constant. Along this path, all properties evolve only as a function of $\varepsilon_{z}$. With $Z\left(\boldsymbol{\varepsilon}, \boldsymbol{\varepsilon}_{m}, \beta\right)$ considered as known, the radial deformation components can be expressed in terms of the axial deformation by using the two equations

$$
\beta p_{c}=\left.\frac{\partial \ln Z}{\partial \varepsilon_{x}}\right|_{\boldsymbol{\varepsilon}_{m}=\boldsymbol{\varepsilon}}=\left.\frac{\partial \ln Z}{\partial \varepsilon_{y}}\right|_{\boldsymbol{\varepsilon}_{m}=\boldsymbol{\varepsilon}}
$$

to obtain the two functions

$$
\varepsilon_{x}=f_{x}\left(\beta, \varepsilon_{z}\right) \quad \text { and } \quad \varepsilon_{y}=f_{y}\left(\beta, \varepsilon_{z}\right)
$$

that are valid only along the load path.

We now write $d U$ in two different ways. First, $d U$ $=\boldsymbol{\tau}: d \boldsymbol{\varepsilon}$ is evaluated along the load path to obtain

$$
d U=\tau_{z} d \varepsilon_{z}-p_{c}\left(d f_{x}+d f_{y}\right) .
$$

Second, we use the fact that $U=U\left(\beta, \boldsymbol{\varepsilon}, \boldsymbol{\varepsilon}_{m}\right)$ to obtain

$$
\begin{aligned}
d U= & \frac{\partial U}{\partial \beta} d \beta+\left(\frac{\partial U}{\partial \varepsilon_{z}}+\frac{\partial U}{\partial \varepsilon_{m z}}\right) d \varepsilon_{z}+\left(\frac{\partial U}{\partial \varepsilon_{x}}+\frac{\partial U}{\partial \varepsilon_{m x}}\right) d f_{x} \\
& +\left(\frac{\partial U}{\partial \varepsilon_{y}}+\frac{\partial U}{\partial \varepsilon_{m y}}\right) d f_{y} .
\end{aligned}
$$

Upon equating Eqs. (45) and (46) we obtain a first-order nonlinear differential equation for $\beta$

$$
a\left(\beta, \varepsilon_{z}\right) \frac{d \beta}{d \varepsilon_{z}}+b\left(\beta, \varepsilon_{z}\right)=0,
$$

where $a$ and $b$ are given by

$$
\begin{gathered}
a=\frac{\partial U}{\partial \beta}+\left(p_{c}+\frac{\partial U}{\partial \varepsilon_{x}}+\frac{\partial U}{\partial \varepsilon_{m x}}\right) \frac{\partial f_{x}}{\partial \beta}+\left(p_{c}+\frac{\partial U}{\partial \varepsilon_{y}}+\frac{\partial U}{\partial \varepsilon_{m y}}\right) \frac{\partial f_{y}}{\partial \beta}, \\
b=-\tau_{z}+\frac{\partial U}{\partial \varepsilon_{z}}+\frac{\partial U}{\partial \varepsilon_{m z}}+\left(p_{c}+\frac{\partial U}{\partial \varepsilon_{x}}+\frac{\partial U}{\partial \varepsilon_{m x}}\right) \frac{\partial f_{x}}{\partial \varepsilon_{z}} \\
+\left(p_{c}+\frac{\partial U}{\partial \varepsilon_{y}}+\frac{\partial U}{\partial \varepsilon_{m y}}\right) \frac{\partial f_{y}}{\partial \varepsilon_{z}} .
\end{gathered}
$$

We are to use $\tau_{z}=-\beta^{-1} \partial \ln Z / \partial \varepsilon_{z}$ and $U=-\partial \ln Z / \partial \beta$ in these expressions for $a$ and $b$ once the function $Z\left(\boldsymbol{\varepsilon}, \boldsymbol{\varepsilon}_{m}, \beta\right)$ has been determined. Furthermore, all partial derivatives are to be evaluated along the load curve; i.e., at $\varepsilon_{m x}$ $=f_{x}\left(\beta, \varepsilon_{z}\right), \varepsilon_{m y}=f_{y}\left(\beta, \varepsilon_{z}\right)$, and $\varepsilon_{m z}=\varepsilon_{z}$.

\section{B. Initial conditions}

In order to integrate Eq. (47), initial conditions must be provided. The initial conditions of our so-called "third law" (i.e., the intact conditions that $\beta=-\infty$ when $\varepsilon_{z}=0$ ) are not well-defined for $\beta$. Thus, Eq. (47) must be integrated not from the intact state, but from a state that contains at least a few cracks so that $\beta \neq-\infty$.

Accordingly, we define "one-crack" initial conditions by considering the point in strain history where on average throughout the ensemble of mesovolumes, there is one crack in each mesovolume. If there are $N$ cells in a mesovolume, the probability of any given cell to be broken somewhere in the ensemble is then $P_{1}=1 / N$. This same probability can also be obtained from Griffith's criterion by integrating the quenched-disorder distribution of Eq. (24) to obtain $P_{1}$ $=\left[\delta E_{1} /\left(\Gamma d^{D-1}\right)\right]^{k+1}$, where $\delta E_{1}=\ell^{D} \boldsymbol{\varepsilon}_{1}: \delta \mathbf{C}: \boldsymbol{\varepsilon}_{1} / 2$ is the elastic energy change due to a single isolated crack and where $\varepsilon_{1}$ is the strain tensor at which on average there is a single crack in each mesovolume. Thus, we have $\boldsymbol{\varepsilon}_{1}: \delta \mathbf{C}: \boldsymbol{\varepsilon}_{1}$ $=2 \Gamma d^{D-1} /\left(N^{1 /(k+1)} \ell^{D}\right)$ that can be used to obtain an expression for the initial axial strain $\varepsilon_{z 1}$ at which on average there is one crack per mesovolume.

To obtain the inverse temperature $\beta_{1}$ corresponding to this initial strain, the exact probability of observing a particular type of crack state is determined and compared to our temperature-dependent Boltzmannian. The particular states 
we choose to analyze are, for simplicity, those having precisely one broken cell.

The probability $p_{j}$ of a state consisting of one broken cell and $N-1$ unbroken cells can be written as

$$
p_{j}=P_{1}\left(1-P_{1}\right)^{N-1} \Pi_{x}[1-\delta P(x)],
$$

where $P_{1}$ is again the probability of having a single broken cell and $\left(1-P_{1}\right)^{N-1}$ is the probability of having $N-1$ broken cells in the absence of other cracks. Thus, the product $\Pi_{x}[1-\delta P(\boldsymbol{x})]$ is the probability that no cells broke due to the strain perturbations caused by the presence of a first broken cell, where $\boldsymbol{x}$ represents distance from this first broken cell. We define $\delta E_{2}(\boldsymbol{x})$ as the elastic energy change in a mesovolume when a second cell breaks solely in the perturbed strain field emanating from a first broken cell. This energy varies with the separation distance $|\boldsymbol{x}|$ between the two cracks as $|\boldsymbol{x}|^{-D}$. We have

$$
\delta P(\boldsymbol{x})=\int_{0}^{\delta E_{2}(\boldsymbol{x})} \pi(e) d e=\left(\frac{\delta E_{2}(\boldsymbol{x})}{\Gamma d^{D-1}}\right)^{k+1}=\frac{c_{2}}{|\boldsymbol{x}|^{D(k+1)}},
$$

where Eq. (24) was used for $\pi$ and where $c_{2}$ depends on both the overall applied strain and the angle from the firstcrack's orientation to the second crack. Since $\delta P$ is small compared to one (restricting to models where cracks are smaller than the cell size $\Lambda$, since the separation distance $|\boldsymbol{x}|$ always exceeds it), we have

$$
\Pi_{x}[1-\delta P(\boldsymbol{x})]=1-\frac{1}{\ell^{D}} \int_{|x|>\Lambda} \frac{c_{2}}{|\boldsymbol{x}|^{D(k+1)}} d^{D} \boldsymbol{x}
$$

and since $k>0$, this spatial integral over the mesovolume can be neglected in the thermodynamic limit.

The conclusion is that

$$
p_{j}=P_{1}\left(1-P_{1}\right)^{N-1}=p_{0} \frac{P_{1}}{1-P_{1}}=p_{0} e^{-\ln (N-1)},
$$

where $p_{0}=\left(1-P_{1}\right)^{N}$ is the probability of the entirely intact state. This can be compared to our probability law where, from Eqs. (26)-(28), we have

$$
p_{j}=p_{0} \exp \left[\beta_{1} \frac{(1-q)}{2} \boldsymbol{\varepsilon}_{1}: \delta \mathbf{C}: \boldsymbol{\varepsilon}_{1}\right] .
$$

Thus, the inverse temperature that holds when $\boldsymbol{\varepsilon}=\boldsymbol{\varepsilon}_{1}$ is

$$
\beta_{1}=-\frac{\ell^{D} N^{1 /(k+1)} \ln (N-1)}{(1-q) \Gamma d^{D-1}} .
$$

\section{Approximate approach to the temperature}

The approach just taken in defining the initial conditions suggests a convenient way of obtaining an approximate expression for the temperature.

Consider "dilute" states $j$ where cracks do not significantly interact. In this case, the probability $P_{m}$ that any one cell has broken when the maximum strain tensor is at $\boldsymbol{\varepsilon}_{m}$ is again just the cumulative distribution $P_{m}$ $=\left[\ell^{D} \boldsymbol{\varepsilon}_{m}: \delta \mathbf{C}: \boldsymbol{\varepsilon}_{m} /\left(2 \Gamma d^{D-1}\right)\right]^{k+1}$. In this case, the probability of observing a noninteracting state $j$ consisting of $\mathcal{N}_{j}$ cracks is $p_{j}=P_{m}^{\mathcal{N}_{j}}\left(1-P_{m}\right)^{\left(N-\mathcal{N}_{j}\right)}$ where we have forgone the analysis of the preceeding section demonstrating that the unbroken-cell probabilities are negligibly influenced by the strain perturbations from the $\mathcal{N}_{j}$ broken cells (at least for $k$ $>0$ ). We may write

$$
p_{j}=p_{0} \exp \left[-\ln \left(\frac{1}{P_{m}}-1\right) \mathcal{N}_{j}\right],
$$

where $p_{0}=\left(1-P_{m}\right)^{N}$ is the probability of the unbroken state.

For such dilute states, the Hamiltonian of Eq. (26) is written (with $\boldsymbol{\varepsilon}_{m}=\boldsymbol{\varepsilon}$ ) as

$$
E_{j}=\frac{1}{2} \boldsymbol{\varepsilon}_{m}: \mathbf{C}_{0}: \boldsymbol{\varepsilon}_{m}-\frac{(1-q)}{2} \boldsymbol{\varepsilon}_{m}: \delta \mathbf{C}: \boldsymbol{\varepsilon}_{m} \mathcal{N}_{j}
$$

so that our probability law predicts

$$
p_{j}=p_{0} \exp \left[\frac{\beta(1-q)}{2} \boldsymbol{\varepsilon}_{m}: \delta \mathbf{C}: \boldsymbol{\varepsilon}_{m} \mathcal{N}_{j}\right] .
$$

Upon using $1 / P_{m}=\left[2 \Gamma d^{D-1} /\left(\ell^{D} \boldsymbol{\varepsilon}_{m}: \delta \mathbf{C}: \boldsymbol{\varepsilon}_{m}\right)\right]^{k+1}$ and equating Eqs. (58) and (56), the temperature is identified

$$
\beta\left(\boldsymbol{\varepsilon}_{m}\right)=\frac{-2 \ln \left\{\left[2 \Gamma d^{D-1} /\left(\ell^{D} \boldsymbol{\varepsilon}_{m}: \delta \mathbf{C}: \boldsymbol{\varepsilon}_{m}\right)\right]^{k+1}-1\right\}}{(1-q) \boldsymbol{\varepsilon}_{m}: \delta \mathbf{C}: \boldsymbol{\varepsilon}_{m}} .
$$

This expression for $\beta$ has the expected behavior that $\beta=$ $-\infty$ when $\boldsymbol{\varepsilon}_{m}=0$, and that $\beta$ is a negative and increasing function of $\boldsymbol{\varepsilon}_{m}$ up to the strain point $P_{m}=1 / 2$ where it smoothly goes to zero. For $P_{m}>1 / 2, \beta$ is a positive and increasing function of $\boldsymbol{\varepsilon}_{m}$. Our probability law with $\beta$ negative predicts the intact state to have the greatest probability, while when $P_{m}>1 / 2$ and $\beta$ is positive, the most probable state jumps to every cell being broken. Although such a phase transition occurs in fiber bundles [24], we demonstrate in Paper III using the exact differential equation for temperature, that the localization transition always occurs prior to this divergent-temperature transition.

We emphasize that Eq. (59) is an approximation to the extent that due to the long-range nature of elastic interactions, one can never truly define a noninteracting state. We use it to obtain an order-of-magnitude idea of the temperature at a given strain. But it should always be considered preferable to obtain the temperature by integrating the exact Eq. (47) from the first-crack (or other exact) initial conditions.

\section{CONCLUSIONS}

The present theory of fracture in disordered solids works from the postulate that the probability $p_{j}$ of observing a mesovolume in a given emergent-crack state $j$ and at a given 
applied strain can be determined by maximizing Shannon's measure of the emergent-crack disorder subject to constraints that come from the energy balance of brittle fracture. These constraints are what allow nonuniform probability distributions to occur. The validity of this postulate can be demonstrated in simpler cases [24] by integrating the probability distribution through history, but its general validity in the case of rocks with interacting cracks remains an open problem. Our approach to answering this question is to use the statistical mechanics that follows from our maximal-disorder postulate to make predictions about the physical properties of real systems and to compare such predictions to laboratory data.

\section{ACKNOWLEDGMENTS}

The authors thank S. Roux and M. Holschneider for useful discussions in the early stages of this work, and D. Lockner for sharing both his data and knowledge of the fracture process. R.T. received financial support from the TMR network "Fractal Structures and Selforganization" through EEC Grant No. FMRXCT980183.
[1] R. L. Blumberg Selinger, Z. G. Wang, W. M. Gelbart, and A. Ben-Shaul, Phys. Rev. A 43, 4396 (1991).

[2] J. B. Rundle and W. Klein, Phys. Rev. Lett. 63, 171 (1989).

[3] Z. G. Wang, U. Landman, R. L. Blumberg Selinger, and W. M. Gelbart, Phys. Rev. B 44, 378 (1991).

[4] L. Golubovič and A. Peredera, Phys. Rev. E 51, 2799 (1995).

[5] P. Meakin, in Statistical Models for the Fracture of Disordered Media, edited by H. Hermann and S. Roux (Elsevier, Amsterdam, 1990).

[6] G. G. Batrouni and A. Hansen, Phys. Rev. Lett. 80, 325 (1998).

[7] L. de Arcangelis and H. J. Herrmann, Phys. Rev. B 39, 2678 (1989).

[8] P. M. Duxbury, P. L. Leath, and P. D. Beale, Phys. Rev. B 36, 367 (1987).

[9] S. Arbabi and M. Sahimi, Phys. Rev. B 47, 695 (1993); M. Sahimi and S. Arbabi, ibid. 47, 703 (1993); 47, 713 (1993).

[10] M. Sahimi and S. Arbabi, Phys. Rev. Lett. 77, 3689 (1996).

[11] H. J. Herrmann, A. Hansen, and S. Roux, Phys. Rev. B 39, 637 (1989).

[12] M. Kloster, A. Hansen, and P. C. Hemmer, Phys. Rev. E 56, 2615 (1997).

[13] A. Hansen and P. C. Hemmer, Phys. Lett. A 184, 394 (1994).

[14] P. C. Hemmer and A. Hansen, J. Appl. Mech. 59, 909 (1992).

[15] S. D. Zhang and E. J. Ding, Phys. Rev. B 53, 646 (1996).
[16] D. Sornette, J. Phys. A 22, L243 (1989).

[17] Y. Moreno, J. B. Gómez, and A. F. Pacheco, Phys. Rev. Lett. 85, 2865 (2000); Physica A 296, 9 (2001).

[18] L. de Arcangelis, A. Hansen, H. J. Herrmann, and S. Roux, Phys. Rev. B 40, 877 (1989).

[19] A. Hansen, E. L. Hinrichsen, and S. Roux, Phys. Rev. B 43, 665 (1991).

[20] Statistical Models for the Fracture of Disordered Media, edited by H. J. Herrmann and S. Roux (North-Holland, Amsterdam, 1990).

[21] S. Zapperi, P. Ray, H. E. Stanley, and A. Vespignani, Phys. Rev. Lett. 78, 1408 (1997).

[22] S. Zapperi, P. Ray, H. E. Stanley, and A. Vespignani, Phys. Rev. E 59, 5049 (1999).

[23] J. V. Andersen, D. Sornette, and K. T. Leung, Phys. Rev. Lett. 78, 2140 (1997).

[24] S. R. Pride and R. Toussaint, Physica A 312, 159 (2002).

[25] J. W. Rudnicki and J. R. Rice, J. Mech. Phys. Solids 23, 371 (1975).

[26] A. A. Griffith, Philos. Trans. R. Soc. London, Ser. A 221, 163 (1920).

[27] C. Shannon, Bell Syst. Tech. J. 27, 373 (1948).

[28] M. S. Paterson, Experimental Rock Deformation-the Brittle Field (Springer-Verlag, Berlin, 1978). 